\title{
Numerical modelling of transonic centripetal flow in radial turbine blade cascade
}

\author{
Petr Straka ${ }^{1, *}$ \\ ${ }^{1}$ VZLU - Czech Aerospace Research Centre, Beranových 130, 19905 Prague 9, Czech Republic
}

\begin{abstract}
Numerical modelling of transonic centripetal turbulent flow in radial blade cascade is described in this paper. Method of the confusor buffer zone is applied to overcome some numerical obstacles related with specifical properties of the outlet confusor. Kinetic energy loss coeficient of the radial blade cascade is compared with its linear representation and with experimental data.
\end{abstract}

\section{Introduction}

Two dimensional flow field in a radial blade cascade is (in case of centripetal flow) limited with specific properties of the outlet confusor. Maximal achievable Mach number on radius of the trailing edges $M_{T E \text {, } \max }$ is limited by the outlet confusor geometry (specifically by the outlet radius $r_{\text {out }}$ ) and by the flow angle on the radius of the trailing edges $\alpha_{T E}$ (which is given by the blade cascade geometry). Relation between $M_{T E \text {, max }}$ and $r_{\text {out }}$ is significant namely for high values of the outlet Mach number. In numerical simulation the outlet confusor is that part of the computational domain, which is bounded with the trailing edges radius $r_{T E}$ and the outlet radius $r_{\text {out }}$. In case of supersonic outlet achieving of higher and higher outlet Mach number is possible only by modification of the outlet confusor geometry - by increasing the outlet radius $r_{\text {out }}$. By other words it means placing the outlet boundary of the computational domain closer and closer to the trailing edges, which may lead to some numerical obstacles related with unphysical shock waves reflections from the outlet boundary.

This paper deals with numerical simulation of transonic centripetal flow through radial blade cascade in range of the outlet Mach number on the trailing edge radius $1.025 \leq M_{T E} \leq$ 1.88 and for the chord based Reynolds number $R e=8.5 \times 10^{5}$. Geometry of the radial blade cascade is derived from the linear blade cascade via conformal mapping as shown in Figure 1. The original linear blade cascade of short axial length was developed for transonic regimes in range of the outlet isentropic Mach number $1.6 \leq M_{\text {out is }} \leq 2.1$ (see [1]).

As mentioned above, achieving high supersonic values of the outlet Mach number in case of centripetal flow is possible only by increasing the outlet radius $r_{\text {out }}$ of the outlet confusor. Beside way of placing the outlet boundary closer and closer to the trailing edges, there is another method called confusor buffer zone proposed in [2]. Method of the confusor buffer zone respects relation between $M_{T E, \max }$ and the outlet confusor geometry and allows

\footnotetext{
*Corresponding author: straka@vzlu.cz
} 
to overcome numerical obstacles related with unphysical shock waves reflections. Method of the confusor buffer zone used in this work is a slight modification of that which is proposed in [2].



Fig. 1. Scheme of the conformal mapping of the linear (left) to the radial (right) blade cascade.

\section{Outlet confusor}

As shown in Figure 2, the outlet confusor is bounded by inlet radius $r_{T E}$ and outlet radius $r_{\text {out }}$. The confusor inlet radius $r_{T E}$ corresponds with the radius over the trailing edges, the outlet radius corresponds with the outlet boundary of the computational domain in numerical simulation.

The flow through the radial confusor was solved in $[3,4]$ theoretically using model of the potential sink-vortex flow. This model is described by system (1) - (2) which is derived from conservation laws of mass, momentum (vortex conservation condition) and energy:

$$
\begin{gathered}
\frac{\partial u_{t}}{\partial r}=-\frac{u_{t}}{r}, \\
\frac{\partial u_{r}}{\partial r}=-\frac{u_{r}}{r} \frac{1+M_{t}^{2}}{1-M_{r}^{2}} .
\end{gathered}
$$

In system (1) - (2) $u_{t}$ and $u_{r}$ are tangential and radial velocity vector components, $M_{t}$ and $M_{r}$ are tangential and radial Mach number components. From (1) it is evidence that in case 


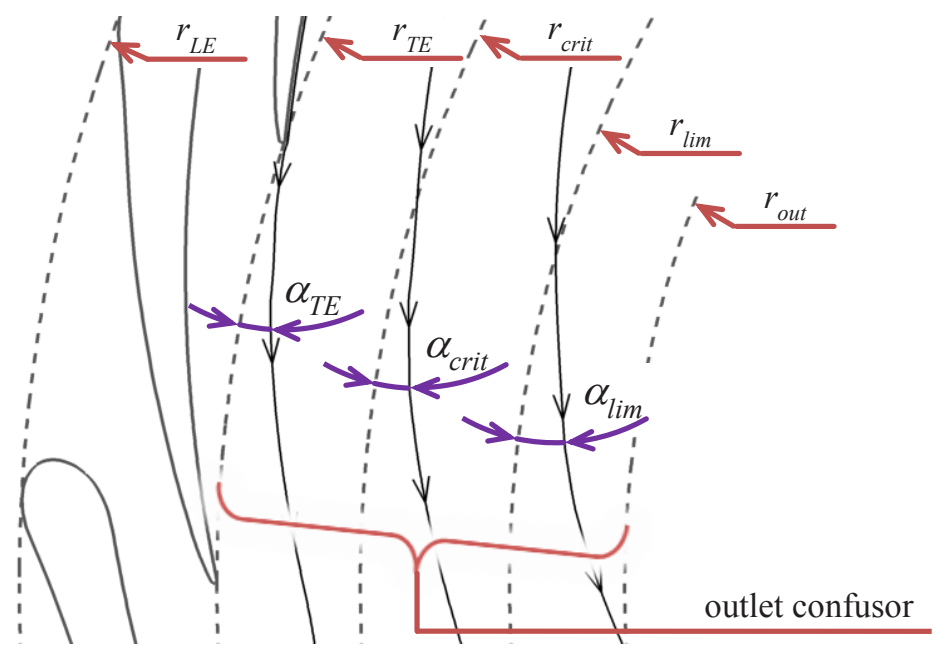

Fig. 2. Scheme of the outlet confusor.

of centripetal flow the tangential velocity component increase towards to the outlet radius $r_{\text {out }}$. Solution of (2) has two branches: for $M_{r}<1$ and for $M_{r}>1$. We will deal with first branch $\left(M_{r}<1\right)$ only in this work. From (2) it is clear that in case of centripetal flow and $M_{r}<1$ also the radial velocity component must increase towards the outlet radius, where limit velocity is given by condition $M_{r} \rightarrow 1$. More details can be found in [3, 4].

An important parameter for solution of flow in radial confusor is the flow angle $\alpha_{\text {crit }}$ at the critical radius (see F 2). Parameter $\alpha_{\text {crit }}$ is characteristic of the cascade exit geometry and is related to the confusor inlet parameters by relation

$$
\tan \alpha_{c r i t}=\left[1+\frac{\kappa-1}{2}\left(1-M_{* T E}^{2}\right)\right]^{\frac{1}{\kappa-1}} \tan \alpha_{T E} .
$$

Note, that relation between the Mach number $M$ and the normalized velocity $M *$ is given as

a)

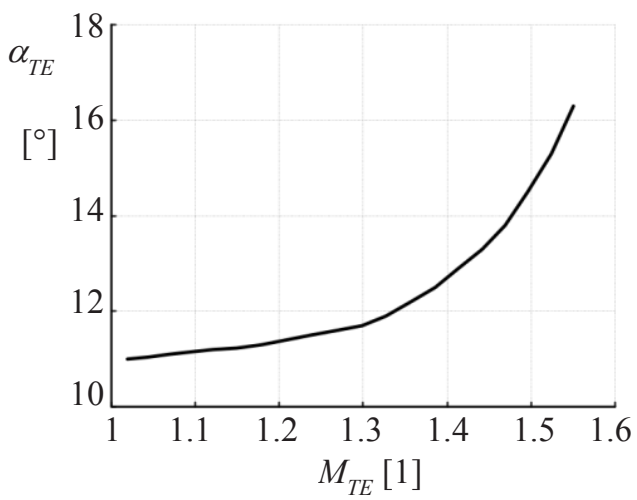

b)



Fig. 3. a) dependency of the flow angle on the Mach number on the trailing edge radius; b) relation between Mach number on the trailing edge radius and the outlet Mach number and the limit Mach number in dependence on the limit radius. 


$$
M_{*}^{2}=\left(\frac{\kappa+1}{2} M^{2}\right) /\left(1+\frac{\kappa-1}{2} M^{2}\right) .
$$

Figure 3a shows dependency of the outlet flow angle on the outlet Mach number for the linear blade cascade. With regard to properties of the conformal mapping, this data can be used in relation (3) for evaluation of parameter $\alpha_{\text {crit }}$.

As mentioned above the condition $M_{r} \rightarrow 1$ defines the limit velocity which can be solved from following system:

$$
\begin{gathered}
M_{* \text { lim }}^{2}=1-\frac{2}{\kappa-1}\left[\left(\frac{\tan \alpha_{c r i t}}{\tan \alpha_{\text {lim }}}\right)^{\kappa-1}-1\right], \\
\tan \alpha_{l i m}=\sqrt{\frac{(\kappa+1)-(\kappa-1) M_{* l i m}^{2}}{(\kappa+1)\left(M_{* l i m}^{2}-1\right)}} .
\end{gathered}
$$

Finally the model of the potential sink-vortex flow defines relation for the limit confusor radius $r_{\text {lim }}$ :

$$
r_{\text {lim }}=r_{T E} / \sqrt{\frac{M_{*_{l i m}}^{2} g\left(M_{*_{l i m}}\right)\left[\tan ^{2} \alpha_{c r i t}+g\left(M_{*_{T E}}\right)\right]}{M_{*_{T E}}^{2} g\left(M_{*_{T E}}\right)\left[\tan ^{2} \alpha_{c r i t}+g\left(M_{*_{l i m}}\right)\right]}},
$$

where $g(M *)$ is given as

$$
g\left(M_{*}\right)=\left[1+\frac{\kappa-1}{2}\left(1-M_{*}^{2}\right)\right]^{\frac{2}{\kappa-1}} .
$$

Relation between the limit Mach number $M_{\text {lim }}$, the limit confusor radius $r_{l i m}$ and the Mach number $M_{T E}$ for presented radial blade cascade is shown in Figure $3 \mathrm{~b}$.

For given outlet radius of the computational domain $r_{\text {out }}$ the Mach number $M_{T E}$ at the

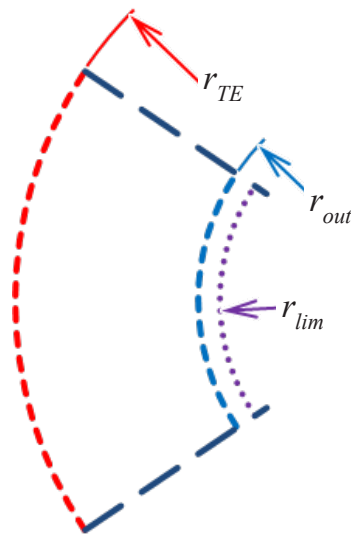

a)

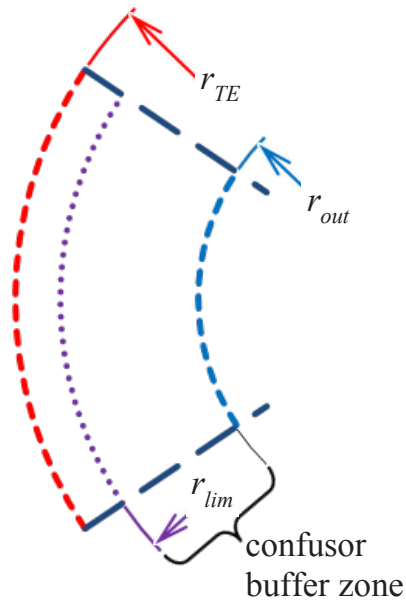

b)

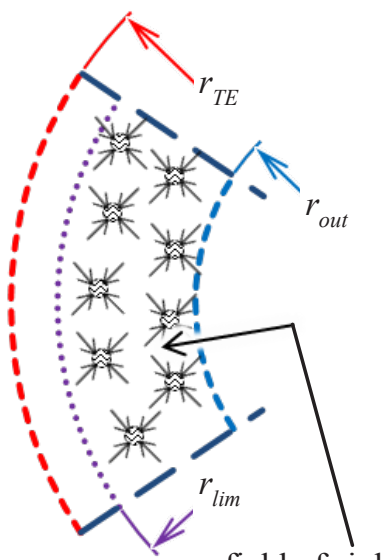

field of sink terms inside the confusor buffer zone

Fig. 4. Scheme of the output confusor; a) case without the buffer zone (limit confusor radius is smaller than the outlet radius); b) case with the buffer zone (limit confusor radius is greater than the outlet radius); c) scheme of the sink terms of the mass inside the confusor buffer zone 
radius $r_{T E}$ can be increased by decreasing of the pressure at the outlet boundary up to limit state given by (5) and (6). The limit state occurs at the outlet radius $r_{\text {out }}$, it means that $r_{\text {out }}=$ $r_{\text {lim }}$. Any further decreasing of the pressure at the outlet boundary (outlet radius) has no effect on flow in radial confusor and consequently in radial blade cascade. Next increasing of the Mach number $M_{T E}$ at radius $r_{T E}$ is possible only through increasing of the limit radius $r_{\text {lim. }}$. It can be done in two ways. One way is to keep the limit state at the outlet boundary $\left(r_{\text {out }}=r_{\text {lim }}\right)$ and increase the outlet radius. This means modifying the computational domain and the computational mesh for each regime. Another way to increase the limit radius is to construct a confusor buffer zone that is bounded by the limit radius and the outlet radius (see middle part of Figure 4). The role of confusor buffer zone is to overcome the limit condition (singularity) given by relation (2) and to avoid above mentioned numerical obstacles related with high supersonic outlet Mach numbers. Construction of such buffer zone using the sink term of the mass will be discussed in following part. The advantage of this approach is that there is no need to modify the computational domain and the computational mesh, because the outlet radius can be fixed for all regimes.

\section{Mathematical models}

Flow in radial blade cascade is modeled as compressible viscous turbulent flow of the perfect gas. Model is described by system of Favre averaged Navier-Stokes equation:

$$
\begin{gathered}
\frac{\partial \rho}{\partial t}+\nabla(\rho \mathbf{U})=q, \\
\frac{\partial(\rho \boldsymbol{U})}{\partial t}+\nabla(\rho \boldsymbol{U} \otimes \boldsymbol{U})+\nabla p=\nabla \tau+q \boldsymbol{U}, \\
\frac{\partial(\rho E)}{\partial t}+\nabla[(\rho E+p) \boldsymbol{U}]=\nabla(\tau \boldsymbol{U})+\nabla(\lambda \nabla T),
\end{gathered}
$$

where $\rho$ is the density, $\mathbf{U}$ is the velocity vector, $p$ is the pressure, $\tau$ is the stress tensor, $E$ is the specific total energy, $\lambda$ is the thermal conductivity and $T$ is the temperature. The system is closed by the equation of state for the perfect gas $p / \rho=r T$ and constant specific heat capacity $c_{p}$ and by two equation nonlinear EARSM $k-\omega$ turbulence model [5].

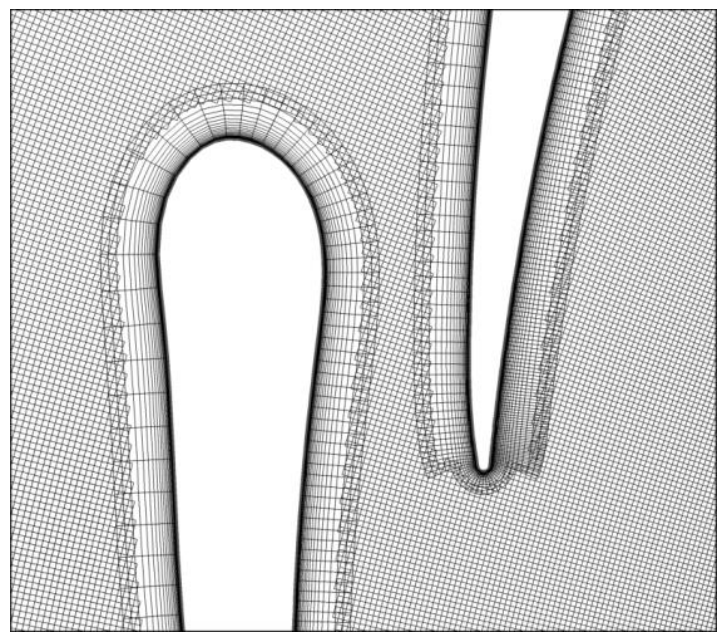

Fig. 5. Detail of the overset "O-H" computational mesh. 
The sink terms of the mass $q$ in equation (9) which is activated inside the confusor buffer zone (see fig. 4) is given as follows:

$$
q=\left\{\begin{array}{rl}
0, & \mathrm{~K} \text { for } r \geq r_{\text {lim }}, \\
-\sigma_{S} \sqrt{2 \rho \Delta p}, & \mathrm{~K} \text { for } r<r_{\mathrm{lim}}
\end{array},\right.
$$

where $r_{\text {lim }}$ is given by relation (7). The pressure difference in (15) is $\Delta p=\max \left(p-p_{\text {out }}, 0\right)$ where $p$ is local pressure and $p_{\text {out }}$ is the outlet pressure. The parameter $\sigma_{S}\left(\mathrm{~m}^{-1}\right)$ controls the mass sink rate. The role of the mass sink term $q$ is to exhaust the area $r<r_{\text {lim }}$ enough to overcome the fact, that the outlet radius $r_{\text {out }}$ is smaller than the limit radius $r_{\text {lim }}$. Contrary to [2] in present work there is used the sink term $q \boldsymbol{U}$ also in equation (10). It means that not only mass is removed from the confusor buffer zone but also the momentum. It prevents unwanted spinning of the flow field inside the confusor buffer zone.

The mathematical model is implemented into the in-house numerical code based on the finite-volume discretization method [6] using the overlapped computational mesh [7] (see Figure 5).

\section{Computation setup}

Solution of flow past presented radial blade cascade was done for the total inlet pressure $114.1 \mathrm{kPa}$, the total inlet temperature $300 \mathrm{~K}$, the inlet turbulence intensity $2 \%$ and radial flow direction. Outlet Mach number $M_{T E}$ at radius $r_{T E}$ is in range $1.025 \leq M_{T E} \leq 1.88$ and the Reynolds number related to outlet velocity and the blade chord length is $R e<8.5 \times 10^{5}$. Because of the lower Reynolds number, the algebraic bypass and separation induced boundary layer transition model [8] is used beside the two-equation turbulence model mentioned in previous section.

Figure 5 shows detail of the overlapped computational mesh which consists from "O"type block over the blade profile and from "H"-type block which cowers periodical section of one pitch. The "O"-type block is refined near the blade surface to ensure that the viscous sublayer is covered at least by five cells. Total number of cells of the computational mesh is 76180 .

Dimension of the outlet boundary is $r_{\text {out }}=135 \mathrm{~mm}$ whereas the trailing edges radius is $r_{T E}=185 \mathrm{~mm}$. Radius of the inlet boundary is $r_{i n}=260 \mathrm{~mm}$ and the leading edges radius is $r_{L E}=209.2 \mathrm{~mm}$.

\section{Results}

Development of the transonic flow field with increasing the value of $M_{T E}$ is shown in Figure 6 in form of numerical reconstruction of the shadow-graph pictures $\left(\nabla^{2} \rho\right)$. The limit radius $r_{\text {lim }}$ is for $M_{T E}=1.025$ identical with radius of the outlet boundary, thus $r_{\text {lim }}=r_{\text {out }}$. Increasing of $M_{T E}$ is possible only by increasing of the radius $r_{\text {lim }}$. This is shown in Figure 6. For Mach number $M_{T E}=1.880$ the limit radius lies $2.5 \mathrm{~mm}$ behind the trailing edges. Note that the limit radius $r_{\text {lim }}$ is the inlet boundary to the confusor buffer zone and that the conditions on the limit radius asymptotically approach state $M_{r} \rightarrow 1$ (see equation (2)). Next, let's note that (according to [3, 4]) the two-dimensional centripetal flow can only exist outside the limit radius. It means that for $r<r_{\text {lim }}$, thus inside the confusor buffer zone, there exists a flow different from the two-dimensional centripetal. That's because the flow field inside the confusor buffer zone is driven by the sink term (12). However, evaluation of aerodynamic and energy properties of the radial blade cascade is possible only outside the limit radius, thus outside the confusor buffer zone. 
In Figure 7 there is depicted the kinetic energy loss coefficient dependence on the outlet Mach number. In the radial blade cascade the kinetic energy loss coefficient was evaluated in distance $2.5 \mathrm{~mm}$ behind the trailing edges. Figure 7 compares CFD prediction of the kinetic energy loss coefficient in radial and linear blade cascades in distance $2.5 \mathrm{~mm}$ behind the trailing edges and CFD prediction of the kinetic energy loss coefficient in linear blade cascade with the experimental data in distance $20 \mathrm{~mm}$ behind the trailing edges. The distance $2.5 \mathrm{~mm}$ behind the trailing edges in case of the radial blade cascade was chosen because flow inside the outlet confusor accelerates and value of the Mach number in a larger distance behind the trailing edges isgnificantly differs from $M_{T E}$ value. Next reason, why it was chosen so little distance behind the trailing edge, is the presence of the confusor buffer zone. The kinetic energy loss coefficient was evaluated using the methodology

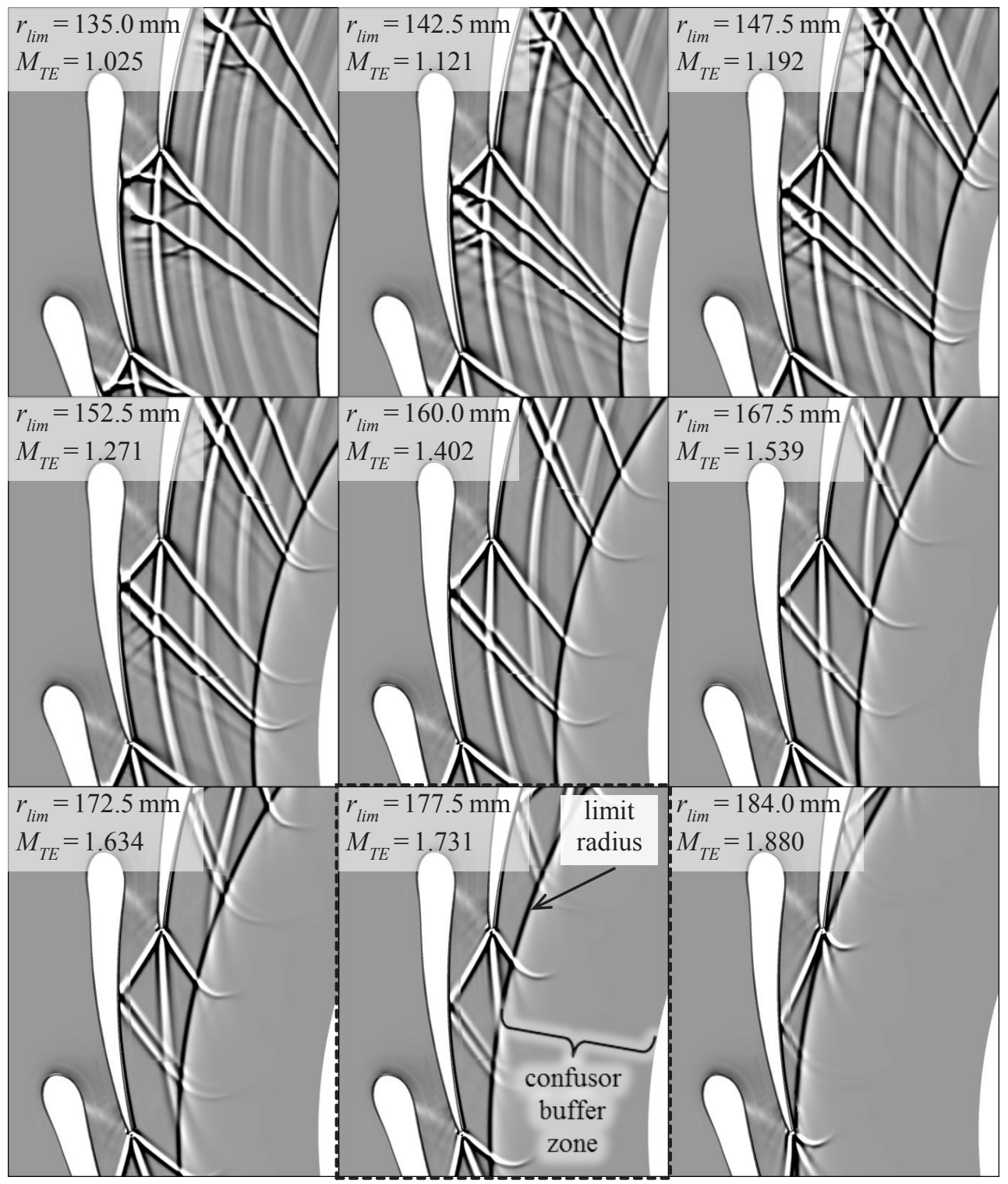

Fig. 6. Numerical shadowgraph reconstruction - development of the transonic flow field depending on the outlet confusor limit radius $r_{l i m}$. 


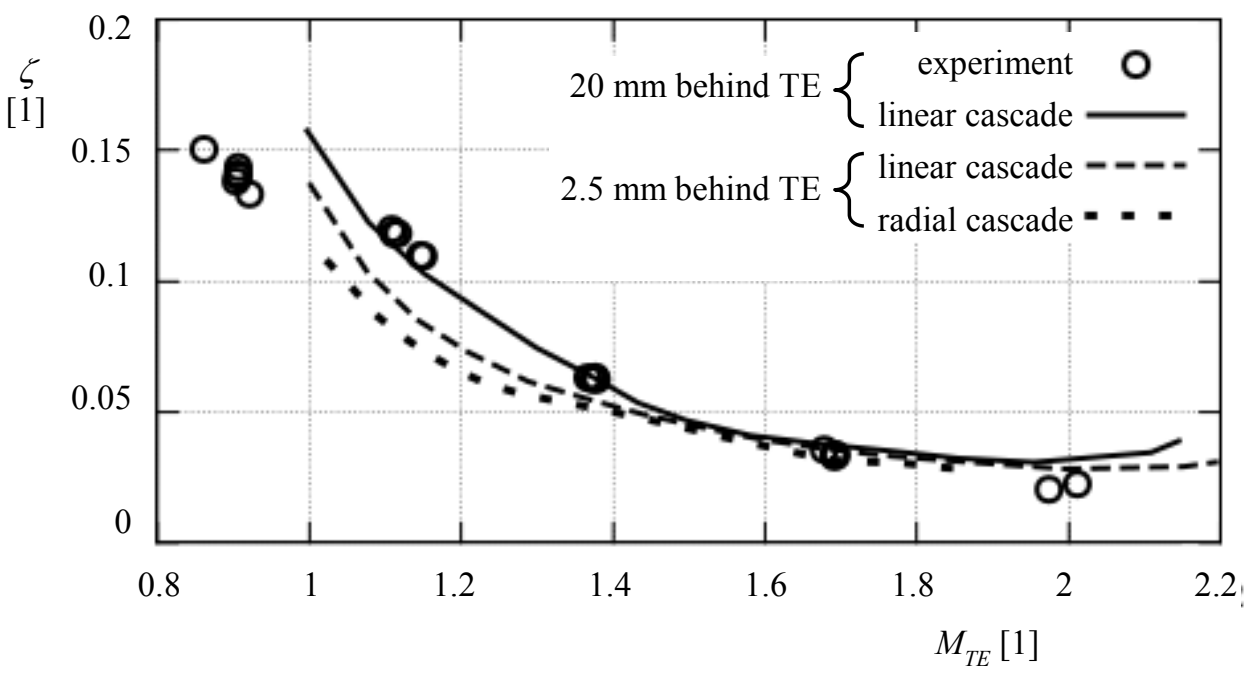

Fig. 7. Dependence of the kinetic energy losses coefficient $\zeta$ on the Mach number $M_{T E}$.

according to [9] but in the arrangement for the polar coordinate system. From Figure 7 it is clear that the kinetic energy loss coefficient of the radial blade cascade is little bit less compared to the linear blade cascade. This is given by the stabilization effect of the accelerated centripetal flow inside the inter-blade channel which slows the growing of the boundary layers thickness. It leads to little bit thinner wake compared to the linear blade cascade.

\section{Conclusions}

Numerical simulations of transonic flow through the radial blade cascade with short radial length were carried out for the outlet Mach numbers $M_{T E}$ in the range from 1.025 up to 1.88. The aerodynamics and energy parameters of the presented radial blade cascade were compared with parameters of the linear blade cascade and with the experimental data.

The confusor buffer zone was proposed to avoid some numerical obstacles related with solution of regimes with high supersonic outlet Mach numbers $M_{T E}$. The main advantage of the confusor buffer zone approach is that there is no need to modify the computational domain and the computational mesh.

This work was supported by the Long-Term Development Concept of Research Organization DKRVO-IPOWER provided by the Ministry of Industry and Trade of the Czech Republic.

\section{References}

1. M. Němec, P. Straka, T. Jelínek, M. Babák, ASME Paper No. GT2010-22490, 1221$1230(2010)$

2. P. Straka, AIP Conf. Proceedings 1889, 020041 (2017)

3. M. Luxa, R. Dvorak, D. Simurda, J. Vimmr, Jour. Thermal Science, 19, 42-46 (2010)

4. M. Luxa, PhD Thesis, Czech Technical University, Prague (2005) (in Czech)

5. A. Hellsten, PhD Thesis, Helsinki University of Technology, Helsinki (2004)

6. P. Straka, VZLÚ Report R-4321, Prague (2008) (in Czech) 
7. P. Straka, Proc. Conf. Finite Volumes for Complex Application VI, Prague, 751-759 (2011)

8. P. Straka, J. Př́ihoda, Proc. Conf. EFM 2010, Liberec, 636-641 (2010)

9. M. Němec, T. Jelínek, M. Mackovič, VZLÚ Report R-5163, Prague (2011) (in Czech) 